\title{
Response Inhibition and Memory Retrieval of Emotional Target Words: Evidence from an Emotional Stop-Signal Task
}

\author{
Cornelia Herbert $^{1}$, Stefan Sütterlin ${ }^{1,2}$ \\ ${ }^{1}$ Department of Psychology, University of Würzburg, Würzburg, Germany \\ ${ }^{2}$ Integrative Research Unit on Social and Individual Development (INSIDE), \\ University of Luxembourg, Luxembourg, Luxembourg \\ E-mail: cornelia.herbert@psychologie.uni-wuerzburg.de \\ Received April 9, 2011; revised May 4, 2011; accepted June 20, 2011
}

\begin{abstract}
Previous research suggests that emotional stimuli capture attention and guide behavior often automatically. The present study investigated the relationship between emotion-driven attention capture and motor response inhibition to emotional words in the stop-signal task. By experimental variations of the onset of motor response inhibition across the time-course of emotional word processing, we show that processing of emotional information significantly interferes with motor response inhibition in an early time-window, previously related to automatic emotion-driven attention capture. Second, we found that stopping reduced memory recall for unpleasant words during a subsequent surprise free recall task supporting assumptions of a link between mechanisms of motor response inhibition and memory functions. Together, our results provide behavioral evidence for dual competition models of emotion and cognition. This study provides an important link between research focusing on different sub-processes of emotion processing (from perception to action and from action to memory).
\end{abstract}

Keywords: Emotion, Response Inhibition, Memory, Motivated Attention

\section{Introduction}

Response inhibition is an important key feature of human behavioral control. Response inhibition includes both, inhibition of anticipated behavioral responses as well as stopping of already initiated behavioral responses to task-relevant stimuli. Regarding emotional stimuli, a wealth of studies suggest that emotional compared to neutral stimuli capture attention and guide behavior automatically [1]. Emotional stimuli are associated with approach and avoidance, i.e., action tendencies that promote individual survival and well-being [1]. Responses to emotional stimuli should thus be generally harder to inhibit compared to neutral stimuli because of their survival relevance. So far, a number of studies support this assumption. Behaviorally, findings converge on a general trend towards reduced performance when confronted with emotional relative to neutral stimuli in tasks that require response inhibition such as the go-nogo task [2-4].
The stop-signal task (SST) [5,6] represents a paradigm in which an already initiated response to task-relevant stimuli has to be inhibited as quickly as possible. The difficulty of response inhibition varies as a function of the temporal delay between the target stimulus and the stopsignal signaling the subject to stop his/her response.

The outcome of this race between responding and stopping has recently been demonstrated to be modulated by the stimulus' emotional significance [7]. Verbruggen and De Houwer [7] used emotional and neutral picture stimuli and the stop-signal task. Pictures were of highly arousing, unpleasant and pleasant content or of low arousing neutral content and presented $500 \mathrm{~ms}$ before the onset of the target stimuli, which consisted of symbolic items (geometric shapes). Reaction times to targets in trials requiring no inhibition (go-trials) were significantly longer for targets preceded by emotional compared to neutral pictures. In addition, processing of emotional compared to neutral pictures significantly increased stop-signal reaction times (SSRT) to the neutral symbolic target 
stimuli.

Although these results strongly support a relationship between emotional processing and response inhibition, the mechanisms that drive this relationship are still unclear. The SST is a behaviorally simple task; nevertheless, performance in the SST can be influenced by many sub-processes. Verbruggen and De Houwer [7] suggested that processing of emotional stimuli influences inhibition of already initiated behavioral responses to task-relevant, but neutral stimuli via attentional mechanisms. Theoretically, the grabbing of attentional resources by emotional stimuli should influence both responding and inhibition of motor responses to task-relevant stimuli, because it leaves less processing resources available for the execution of processes and actions that one is engaged in while the emotional stimulus is processed [1].

Concerning visual processing of emotional stimuli electrophysiological studies examining the time-course of emotional stimulus processing propose that attention capture by emotional stimuli occurs within two consecutive time windows: A first time window starting as early as $200 \mathrm{~ms}$ after emotional stimulus-onset and a second, later time-window associated with more elaborate and controlled processing of emotional stimuli [8-11]. Earlier modulation $<200$ ms post stimulus-onset attributable to stimulus-driven attention by emotional content has also occasionally been reported, even with more symbolic stimuli such as emotional words [12].

Taking these findings into account one may hypothesize effects of emotion on response inhibition to occur in time-windows much earlier than those examined by Verbruggen and De Houwer [7], where the temporal delay between the onset of a picture and the stop-signal was greater than $500 \mathrm{~ms}$. Second, these effects should not be restricted to picture stimuli of emotional content, but principally also occur when emotional content is conveyed by language stimuli $[8,9]$.

The present study aimed to provide direct evidence for these assumptions. Contrary to previous research [7] in this study emotional and neutral words were used as targets of response inhibition in the SST and response inhibition to emotional and neutral words was investigated within the first $150 \mathrm{~ms}$ - $250 \mathrm{~ms}$ post stimulus onset. Based on the findings reported above, we expected longer reaction times to emotional compared to neutral words on trials where no stop-signal is presented (go- trials). In line with this, we expected stop-signal reaction time (SSRT) to be increased for emotional compared to neutral words indicating harder response inhibition to emotional than neutral targets. Above all, we expected this emotional-neutral SSRT effect to vary as a function of the stimulus onset asynchrony (SOA) of the stop-signal SOA and to interact with the emotional valence of the words if processes required for successful response inhibition are influenced by the early attention grabbing power of the emotional target words.

A second issue addressed in the present study relates to recent findings on a postulated association between mechanisms supporting successful motor response inhibition and memory. The need to control behavior is not limited to inhibition of overt behavioral reactions but on many occasions affords inhibition of unwanted cognitions including unpleasant memories from coming to mind $[13,14]$. Neuroimaging studies on response inhibition demonstrate enhanced activation of the dorsal and ventral prefrontal cortex $[15,16]$, critical for executive control and behavioral regulation. Recent research by Anderson and colleagues [17] suggested that activation in prefrontal executive control systems mediates hippocampal activity and memory of unpleasant stimuli that participants were asked to suppress during stimulus exposure. One influential hypothesis derived from these studies is that the potential mechanisms underlying memory inhibition are analogous to those controlling overt behavior in reaction time tasks. If this assumption is correct, to be stopped items (in the stop-signal trials) should be spontaneously remembered less frequently than to be responded items (in the go-trials), in particular when their meaning is associated with unpleasant valence.

Based on the above reported literature the aims of the present study can be summarized as follows: a) to examine the extent to which the emotional valence of a target stimulus influences responding and motor response inhibition in the SST at short latencies, b) to explore whether memory retrieval of emotional targets is affected by processes related to motor response inhibition.

\section{Methods}

\subsection{Participants}

Thirty-one healthy adult students (13 males, 18 females, $\mathrm{M}=24.0$ years, $\mathrm{SD}=3.0$ ), all right-handed native speakers of German participated in the study. Participants received course credit and gave written informed consent to participate in the study. Written informed consent was given in accordance with the ethical princeples of the Declaration of Helsinki. Only participants, who reported to be in good health (i.e., no current or history of drug abuse, chronic physical conditions, neurological diseases, mental ill health) and with normal sense of hearing and normal or corrected to normal vision were recruited. Participants had normal state, $\mathrm{M}=39.0, \mathrm{SD}=$ 7.8, and trait anxiety scores, $\mathrm{M}=35.9, \mathrm{SD}=8.7$, on the Spielberger State and Trait Anxiety Inventory (STAI) [18] and reported more positive than negative mood (po- 
sitive affect: $\mathrm{M}=28.6, \mathrm{SD}=6.62$; negative affect: $\mathrm{M}=$ $14.29, \mathrm{SD}=6.49$ ) on the PANAS, positive affect, negative affect scales [19].

\subsection{Stimulus Material}

Experimental stimuli were 50 pleasant, 50 unpleasant, and 50 neutral nouns which comprised on average six characters and according to the CELEX data base [20] were frequently used in German. Nouns were taken from a word database previously collected by our own research group ${ }^{1}$ that provides for each word mean valence, arousal, and concreteness ratings of an independent sample of adult native speakers with comparable backgrounds and ages to the participants of the present study. Valence and arousal ratings were obtained on the Self Assessment Manikin (SAM) [21], a culture-free, non-verbal rating procedure. Concreteness ratings were obtained on a ninepoint SAM-like scale. Mean valence, arousal and concreteness scores as well as word length and word frequency counts of the words are listed in Table 1. Pleasant and unpleasant nouns did not differ significantly in emotional arousal, but were both significantly more arousing than neutral nouns. Mean valence ratings differed as expected (pleasant $>$ neutral $>$ unpleasant). There were no significant differences for concreteness, word length, or word frequency across the three emotional categories (all $p>0.2$ ).

Stop-signal task: The SST was presented on a 19-inch computer display using Presentation software (Neurobehavioral Systems Inc.). Nouns were presented in black letters (font $=$ "Times"; size $=40$ ) centred on a white background, the viewing distance from the screen was $80-90 \mathrm{~cm}$. Half of the nouns of each category were assigned to the go-trials, the other half to the stop-trials. Assignment of words to trials (go and stop) was randomized and counterbalanced across participants. Go-trials were repeated 4 times; stop-trials two times resulting in a total of 450 trials (75\% Go, 25\% Stop). Nouns were presented for $500 \mathrm{~ms}$ in each of the trials and followed by an interstimulus-interval in which a fixation cross was displayed for $1200 \mathrm{~ms}$ - $1500 \mathrm{~ms}$ in the middle of the screen. In the stop-trials nouns were followed by an acoustic stop-signal $(1000 \mathrm{~Hz}, 500 \mathrm{~ms}$, sound pressure level 80 $\mathrm{dB}$ ) prompting participants to stop their response. Stimulus onset asynchrony between the acoustic stop-signal and noun-onset was $150 \mathrm{~ms}, 200 \mathrm{~ms}$ and $250 \mathrm{~ms}$, respectively. Each of the three stop-signal SOAs occurred equally often. Go- and stop-trials with different SOAs were presented randomly such that repeated words were

\footnotetext{
${ }^{1}$ The complete list of words used in this study (original and translation) together with valence and arousal ratings is available from the authors upon request.
}

Table 1. Stimulus material characteristics.

\begin{tabular}{cccc}
\hline & Unpleasant & Pleasant & Neutral \\
\hline Valence & $2.63(0.13)$ & $7.33(0.12)$ & $5.31(0.07)$ \\
Arousal & $5.07(0.12)$ & $5.03(0.10)$ & $2.32(0.08)$ \\
Concreteness & $4.17(0.25)$ & $4.78(0.22)$ & $4.20(0.32)$ \\
Word length & $6.20(0.26)$ & $6.96(0.35)$ & $6.88(0.22)$ \\
Word frequency & $97.34(31.35)$ & $144.92(24.01)$ & $129.96(19.79)$ \\
\hline
\end{tabular}

Note: Mean valence, arousal and concreteness scores range from 1 (extremely negative valence, extremely low arousal or concreteness) to 9 (extremely positive valence, extremely high arousal or concreteness). Word frequency counts for written language are based on the standardized worddatabase CELEX [20]. Word length represents number of letters. Standard errors are in parentheses.

never consecutive to avoid stimulus repetition induced by short-term response time adjustment effects time adjustment effects [22]. Presentation sequence of words from the three different word categories shared convergence in probability (i.e., each word of a category had the same probability to be followed by a word of the same or a different word category).

\subsection{Procedure}

Upon arrival at the laboratory participants were seated in a comfortable chair, gave written informed consent, they were questioned about their health and filled in the selfreport questionnaires on mood (PANAS) [19], and state and trait anxiety (STAI) [18]. Participants were given detailed instruction concerning the SST. They were told to respond to the words as quickly as possible by pressing a response key with the index finger of the right hand. They were also told that on some trials a tone will occur signalizing the stop-trial and the requirement to stop the response to the target stimuli. Participants were asked not to wait for the tone and respond to the targets as quickly as possible. After the completion of the SST, participants were instructed to recall as many of the presented words as they could remember in a surprise free recall test and were debriefed about the purpose of the present study.

\subsection{Data Reduction and Statistical Analyses}

Stop-signal task: Stop-signal reaction time (SSRT) and percentage of correctly suppressed responses (stop-signal trials) were recorded and analyzed separately for the three SOAs and word categories. Stop-signal reaction time was calculated according to the algorithm proposed by Logan [5,6], where SSRT is derived from the distribution of response times to the go-trials, the observed probability of responding in the stop-signal trials and the stop-signal delay. Reaction time data (go-trials) and per- 
centage of correctly responded go-trials were analyzed with repeated measurements of variance (ANOVA) containing the factor "Valence" (unpleasant, pleasant, and neutral) as within subject factor. SSRT and number of correctly inhibited stop-trials were analyzed with repeated measurements of variance (ANOVA) containing the factors "Valence" (unpleasant, pleasant, and neutral) and "SOA" (SOA150, SOA200, and SOA250) as within subject factors.

Memory Data-Free recall task: Participants' memory performance was analyzed with an ANOVA design containing the factors "Trials" (go and stop) and "Valence" (unpleasant, pleasant, and neutral) as within subject factors. Only correctly remembered words on successfully to be responded and to be stopped trials were entered into the analysis. For all measures reported above, significant main effects and interaction effects were tested with post hoc paired sample $t$-tests. $p$-values were corrected using the Bonferroni adjustment.

\section{Results}

Stop-signal task: Participants responded on average correctly in $96 \%$ of the go-trials, regardless of whether nouns were of unpleasant, $\mathrm{M}=96.64, \mathrm{SD}=11.21$, pleasant, $\mathrm{M}$ $=96.06, \mathrm{SD}=11.08$, or neutral meaning, $\mathrm{M}=96.54, \mathrm{SD}$ $=11.32$. Reaction times to successfully re- sponded go-trials differed significantly as a function of word valence, "Valence": $F(2,60)=4.18, p<0.01$. Re- action times in go-trials were significantly longer for unpleasant and pleasant compared to neutral nouns (unpleasant: $\mathrm{M}=$ 421.0, SD = 66.5; pleasant: $\mathrm{M}=418.5$, $\mathrm{SD}=62.6$; neutral: $\mathrm{M}=414.3$, $\mathrm{SD}=61.5$ ), but did not differ significantly between unpleasant and pleasant nouns (unpleasant - pleasant: $t(30)=-1.069, p=0.29$ ).

Number of correctly inhibited stop-trials varied significantly with the SOA of the stop-signal, $F(2,60)=$ 48.15, $p<0.001$. On average, participants responded more often to stop-trials the longer the SOA of the stopsignal, demonstrating the increased difficulty of motor response inhibition the longer the SOA of the stop-signal [5]. Rate of correctly inhibited stop-trials was 73\% at SOA150 and close to 50\% at both SOA200 (59\%) and SOA250 (45\%), confirming SSRT as a reliable measure of response inhibition [23]. Insignificant interaction effects of the factors "Valence x SOA" showed that this was true for emotional as well as neutral nouns, "Valence x SOA": $F(4,120)=0.75, p=0.5$.

Stop-signal reaction time varied with the stop-signal, "SOA": $F(2,60)=10.47, p<0.001$ and, in addition, showed a significant interaction of the factors "SOA" and "Valence", $F(4,120)=2.5, p<0.05$. For SOA250, SSRT differed significantly between emotional and neu- tral nouns: SSRT was significantly enhanced for emotional compared to neutral nouns (unpleasant-neutral: $t(30)=3.14, p<0.01$; pleasant-neutral: $t(30)=2.24, p<$ 0.05). Results are summarized in Table 2 and displayed in Figure 1.

Memory Data-Free recall task: Emotional nouns, unpleasant and pleasant, were significantly better remembered than neutral nouns (see Figure 2), indicating enhanced memory performance for emotional compared to neutral items on later retrieval, "Valence": $F(2,16)=18.3$, $p<0.01$. Significant interactions of the factors "Valence $x$ Trial" and post hoc tests of the interaction effect, however, demonstrated that unpleasant nouns were remembered less frequently when the corresponding responses to them were successfully inhibited, "Valence $\mathrm{x}$ Trial": $F(2,60)=4.07, p<0.05$; unpleasant: $t(30)=2.40, p<$ 0.01 .

\section{Discussion}

This study investigated response inhibition to emotional and neutral target words in the stop-signal task at three discrete stop-signal delays (SOA150, SOA200, SOA250) and examined memory for emotional and neutral target words as a function of response inhibition. Based on the literature on the effects of emotion on attention [8-10, 24], we expected reaction times to emotional compared to neutral words to indicate attention capture by emotional relative to neutral words. We further hypothesized attention capture by emotional stimuli to interfere with response inhibition across the different SOAs. Processing of unpleasant and pleasant nouns produced significantly longer reaction times on go-trials compared to neutral nouns. Stop-signal reaction time, as an index of response inhibition, varied significantly as a function of both the emotional valence of the words and the stop-signal delay. For neutral nouns SSRT decreased from SOA150 to SOA250. This decrease in SSRT is a commonly reported pattern (for an overview see [5]). But for emotional nouns

Table 2. Stop-signal reaction times (SSRT) and reaction times in go-trials.

\begin{tabular}{cccc}
\hline Stop-signal task (SST) & unpleasant & pleasant & neutral \\
\hline Reaction times (go-trials) & $421.0(66.5)$ & $418.5(62.6)$ & $414.3(61.5)$ \\
SSRT (stop-trials) & & & \\
SOA150 & $213.6(34.8)$ & $203.8(51.1)$ & $207.1(41.4)$ \\
SOA200 & $188.2(31.2)$ & $184.2(34.8)$ & $186.6(33.2)$ \\
SOA250 & $192.9(44.2)$ & $185.0(51.7)$ & $169.7(37.4)$ \\
\hline
\end{tabular}

Note. Stop-signal performance including reaction times in go-trials and SOA dependent SSRT as a measure of successful stopping and response inhibition; measures are listed separately for unpleasant, pleasant and neutral words. Standard deviations are in parentheses. 


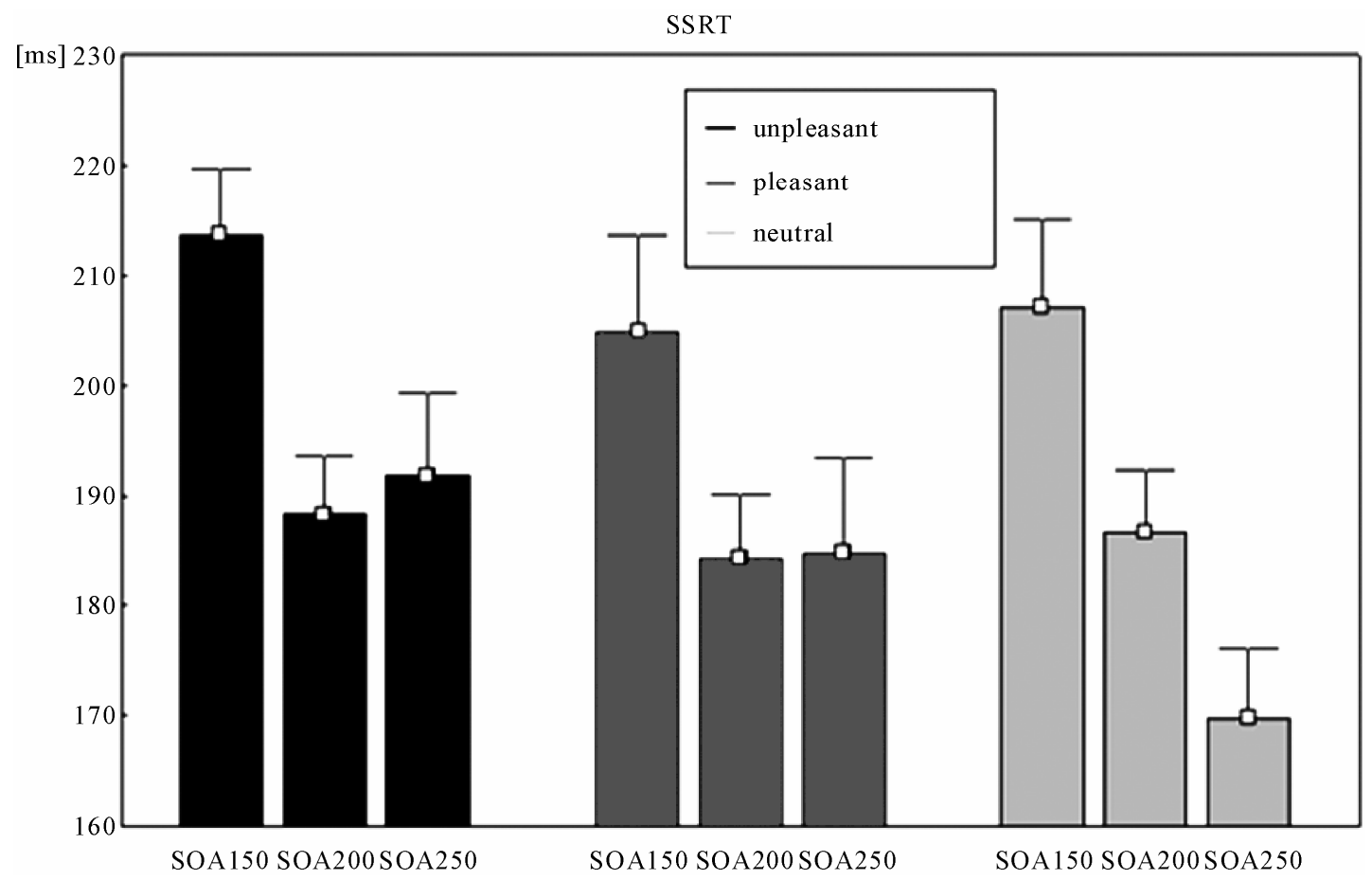

Figure 1. Modulation of SSRT by stimulus valence and SOA (mean and standard errors).

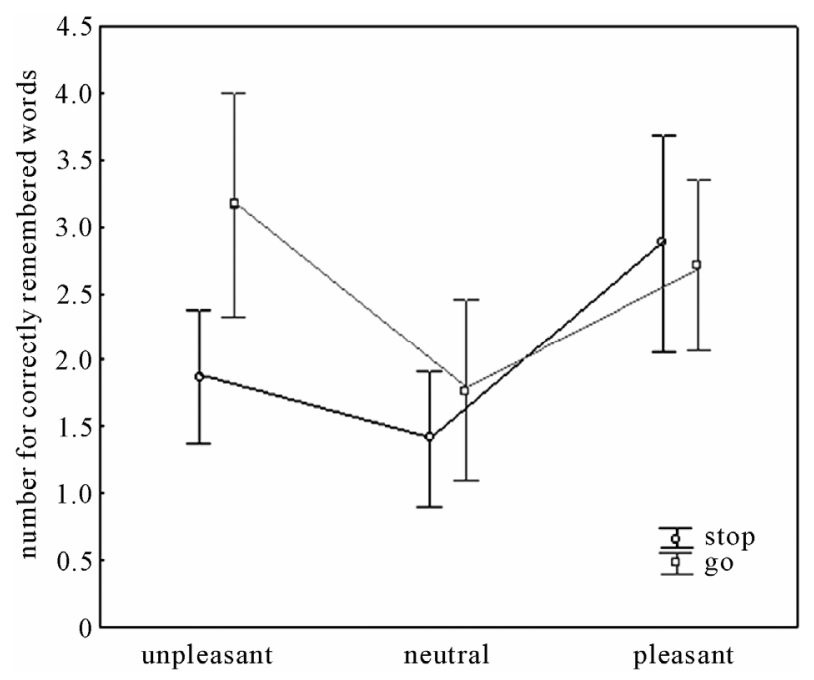

Figure 2. Memory performance for emotional and neutral nouns.

the decrease in SSRT across SOAs did not appear. For emotional nouns relative to neutral nouns SSRT was significantly increased at SOA250.

Previously, it has been speculated [7] that processing of emotional stimuli interferes with response inhibition because emotional stimuli capture more attentional resources compared to neutral stimuli and therefore less processing resources are available for successful inhibittion of motor responses to the target stimuli. The results of the present study provide further evidence in favor of this speculation and suggest that attention capture by emotional stimuli interacts with response inhibition at even earlier time points than those examined previously. Viewed from a biological perspective, such early effects of emotion on response inhibition as reported in the present study support dual competition models of cognition, emotion and motivation [25] that propose closely interrelated and dependent processes involved in emotion perception and response inhibition. Neurally, these effects could be accomplished by fronto-limbic connections biasing signal transfer from sensory association cortex to prefrontal cortex and vice versa [25].

Our observation of an inhibition-dependent memory effect for emotionally unpleasant targets is also in line with dual competition models. Consistent with several laboratory studies on emotional memory [26], emotional nouns were post-experimentally better remembered than neutral words. However, when free recall performance was analyzed as a function of response inhibition, to be stopped items were remembered less frequently than to be responded items when their meaning was of negative content. Anderson and colleagues $[13,17]$ recently demonstrated inhibition-related effects on later memory retrieval for unpleasant stimuli that participants were asked to inhibit and suppress during stimulus exposure. Based on neuroimaging findings [17], the authors proposed a potential link between processes associated with memory inhibition and inhibition of (motor) responses to unpleasant targets. To our knowledge, the present study is 
the first to examine this hypothesis directly by using a motor response inhibition task and both emotional (unpleasant and pleasant) and neutral stimuli as targets of response inhibition. Regarding unpleasant words, our findings support the assumption of a relationship between memory and inhibitory processes related to motor response inhibition [13,14,17]. Interestingly, stopping had no influence on recall of pleasant items. This also extends previous research, as this study, contrary to the past memory inhibition studies cited above, made use of stimuli of unpleasant as well as pleasant and neutral valence. Although the reasons for the not impaired memory retrieval of positive target stimuli requires future research, the finding fits nicely with everyday life experience: that attempts not to respond to pleasurable or rewarding cues (e.g., palatable food when on diet, expensive clothes when short of money, etc.) does not necessarily weaken the representation of these cues in memory.

In summary, our results provided evidence that processing and response inhibition of emotional targets interact. These interaction effects appear to arise even at very short latencies of $250 \mathrm{~ms}$ after stimulus onset, i.e., in a time-window, where according to the previous lit- erature early attention capture by emotional stimuli is expected to be most pronounced. In addition, our results support a relationship between emotion-modulated motor response inhibition and emotional memory. Future studies may offer further insight into the interplay between the mechanisms supporting and attenuating response and memory inhibition in emotion-laden contexts. Application of this experimental approach to clinical samples (e.g., drug addiction, eating disorder, or attention deficit hyperactivity disorder) might be especially interesting to this end [27].

\section{Acknowledgements}

This research was supported by the German Research Foundation (DFG).

\section{References}

[1] P. J. Lang, M. M. Bradley and B. N. Cuthbert, "Motivated Attention: Affect, Activation, and Action,” In: P. J. Lang, R. F. Simons and M. Balaban, Eds., Attention and Emotion: Sensory and Motivational Processes, Erlbaum, Mahwah, 1997, pp. 97-135.

[2] J. DeHouwer and H. Tibboel, "Stop What You Are Not Doing! Emotional Pictures Interfere with the Task Not to Respond,” Psychonomic Bulletin \& Review, Vol. 17, No. 5, 2010, pp. 699-703. doi:10.3758/PBR.17.5.699

[3] M. Goldstein, G. Brendel, O. Tuescher, H. Pan, J. Epstein, et al., "Neural Substrates of the Interaction of Emotional Stimulus Processing and Motor Inhibitory Control: An
Emotional Linguistic Go/No-Go fMRI Study,” Neuroimage, Vol. 36, No. 3 2007, pp. 1026-1040. doi:10.1016/j.neuroimage.2007.01.056

[4] K. P. Schulz, J. Fan, O. Magidina, D. J. Marks, B. Hahn, et al., "Does the Emotional Go/No-Go Task Really Measure Behavioral Inhibition? Convergence with Measures on a Non-Emotional Analog," Archives of Clinical Neuropsychology, Vol. 22, No. 2, 2007, pp. 151160. doi:10.1016/j.acn.2006.12.001

[5] G. D. Logan, "On the Ability to Inhibit Thought and Action: A User's Guide to the Stop-Signal Paradigm,” In: D. Dagenbach and T. H. Carr, Eds., Inhibitory Processes in Attention, Memory, and Language, Academic Press, San Diego, 1994, pp. 184-239.

[6] G. D. Logan and W. B. Cowan, "On the Ability to Inhibit Thought and Action: A Theory of an Act of Control," Psychological Review, Vol. 91, No. 3, pp. 295-327. doi:10.1037/0033-295X.91.3.295

[7] F. Verbruggen and J. De Houwer, "Do Emotional Stimuli Interfere with Response Inhibition? Evidence from the Stop Signal Paradigm," Cognition \& Emotion, Vol. 21, No. 2, 2007, pp. 391-403. doi:10.1080/02699930600625081

[8] C. Herbert, M. Junghofer and J. Kissler, "Event Related Potentials to Emotional Adjectives during Reading," Psychophysiology, Vol. 45, No. 3, 2008, 487-498. doi:10.1111/j.1469-8986.2007.00638.x

[9] J. Kissler, C. Herbert, P. Peyk and M. Junghofer, "Buzzwords: Early Cortical Responses to Emotional Words during Reading," Psychological Science, Vol. 18, No. 6, 2007, pp. 475-480. doi:10.1111/j.1467-9280.2007.01924.x

[10] H. T. Schupp, T. Flaisch, J. Stockburger and M. Junghofer, "Emotion and Attention: Event-Related Brain Potential Studies," Progress in Brain Research, Vol. 156, 2006, pp. 31-51. doi:10.1016/S0079-6123(06)56002-9

[11] J. Kissler, R. Assadollahi and C. Herbert, "Emotional and Semantic Networks in Visual Word Processing: Insights from ERP Studies," Progress in Brain Research, Vol. 156, 2006, pp. 147-183. doi:10.1016/S0079-6123(06)56008-X

[12] M. J. Hofmann, L. Kuchinke, S. Tamm, M. L. Vo and A. M. Jacobs, "Affective Processing within 1/10th of a Second: High Arousal Is Necessary for Early Facilitative Processing of Negative but not Positive Words," Cognitive Affective and Behavioral Neuroscience, Vol. 9, No. 4, 2009, pp. 389-397. doi:10.3758/9.4.389

[13] M. C. Anderson and C. Green, "Suppressing Unwanted Memories by Executive Control,” Nature, Vol. 410, 2001, pp. 366-369. doi:10.1038/35066572

[14] M. C. Anderson and B. C. Levy, "Suppressing Unwanted Memories," Current Directions in Psychological Science, Vol. 18, No. 4, 2009, pp. 189-194. doi:10.1111/j.1467-8721.2009.01634.X

[15] S. Konishi, K. Nakajima, I. Uchida, H. Kikyo, M. Kameyama, et al., "Common Inhibitory Mechanisms in Human Inferior Prefrontal Cortex Revealed by Functional MRI,” Brain, Vol. 122, No. 5, 1999, pp. 981-991. 
doi:10.1093/brain/122.5.981

[16] C. R. Li, C. Huang, R. T. Constable and R. Sinha, "Imaging Response Inhibition in a Stop-Signal Task: Neural Correlates Independent of Signal Monitoring and PostResponse Processing," The Journal of Neuroscience, Vol. 26, No. 1, 2006, pp. 186-192. doi:10.1523/JNEUROSCI.3741-05.2006

[17] M. C. Anderson, K. N. Ochsner, B. Kuhl, J. Cooper, E. Robertson, S. W. Gabrieli, et al., "Neural Systems Underlying the Suppression of Unwanted Memories," Science, Vol. 303, 2004, pp. 232-235. doi:10.1126/science.1089504

[18] L. Laux, P. Glanzmann, P. Schaffner and C. D. Spielberger, "Das State-Trait-Angstinventar (STAI)," Beltz, Weinheim, 1981.

[19] D. Watson, L. A. Clark and A. Tellegen, "Development and Validation of Brief Measures of Positive and Negative Affect: The PANAS Scales,” Journal of Personality and Social Psychology, Vol. 54, No. 6, 1988, pp. 10631070. doi:10.1037/0022-3514.54.6.1063

[20] R. H. Baayen, R. Piepenbrock and L. Gulikers, "The CELEX Lexical Database (CD-ROM)," Linguistic Data Consortium, University of Pennsylvania, Philadelphia, 1995.

[21] M. M. Bradley and P. J. Lang, "Measuring Emotion: The Self-Assessment Manikin and the Semantic Differential," Journal of Behavioral Therapy and Experimental Psychiatry, Vol. 25, No. 1, 1994, pp. 49-59.

\section{doi:10.1016/0005-7916(94)90063-9}

[22] F. Verbruggen and G. D. Logan, "Long-Term Aftereffects of Response Inhibition: Memory Retrieval, Task Goals, and Cognitive Control,” Journal of Experimental Psychology: Human Perception and Performance, Vol. 34, No. 5, 2008, pp. 1229-1235. doi:10.1037/0096-1523.34.5.1229

[23] G. P. H. Band, M. W. van der Molen and G. D. Logan, "Horse-Race Model Simulations of the Stop-Signal Procedure,” Acta Psychologica, Vol. 112, No. 2, 2003, pp. 105-142. doi:10.1016/S0001-6918(02)00079-3

[24] J. A. Hinojosa, C. Méndez-Bértolo and M. A. Pozo, "Looking at Emotional Words Is Not the Same As Reading Emotional Words: Behavioral and Neural Correlates,” Psychophysiology, 2010. http://onlinelibrary.wiley.com/doi/10.1111/j.1469-8986.2 010.00982.x/full

[25] L. Pessoa, "How Do Emotion and Motivation Direct Executive Control?” Trends in Cognitive Science, Vol. 13, No. 4, 2009, pp. 160-166. doi:10.1016/j.tics.2009.01.006

[26] M. M. Bradley, "Emotional Memory: A Dimensional Analysis, ” In: S. H. M. van Goozen, N. E. van de Poll and J. A. Sergeant, Eds., Emotions: Essays on Emotion Theory, Erlbaum, Hillsdale, 1994, pp. 97-134.

[27] F. Verbruggen and G. D. Logan, "Response Inhibition in the Stop-Signal Paradigm,” Trends in Cognitive Science, Vol. 12, No. 11, 2008, pp. 418-424. doi:10.1016/j.tics.2008.07.005 